ARTICLE

https://doi.org/10.1038/s41467-019-08958-9

\title{
Unveiling the operation mechanism of layered perovskite solar cells
}

Yun Lin', Yanjun Fang ${ }^{1}$, Jingjing Zhao (1) ${ }^{1}$, Yuchuan Shao ${ }^{2}$, Samuel J. Stuard (1) ${ }^{3}$, Masrur Morshed Nahid ${ }^{3}$, Harald Ade ${ }^{3}$, Qi Wang ${ }^{2}$, Jeffrey E. Shield ${ }^{1,4}$, Ninghao Zhou ${ }^{5}$, Andrew M. Moran ${ }^{5}$ \& Jinsong Huang (1) ${ }^{1,2}$

Layered perovskites have been shown to improve the stability of perovskite solar cells while its operation mechanism remains unclear. Here we investigate the process for the conversion of light to electrical current in high performance layered perovskite solar cells by examining its real morphology. The layered perovskite films in this study are found to be a mixture of layered and three dimensional (3D)-like phases with phase separations at micrometer and nanometer scale in both vertical and lateral directions. This phase separation is explained by the surface initiated crystallization process and the competition of the crystallization between 3D-like and layered perovskites. We further propose that the working mechanisms of the layered perovskite solar cells involve energy transfer from layered to 3D-like perovskite network. The impact of morphology on efficiency and stability of the hot-cast layered perovskite solar cells are also discussed to provide guidelines for the future improvement.

\footnotetext{
${ }^{1}$ Department of Mechanical and Materials Engineering, University of Nebraska-Lincoln, Lincoln, NE 68588, USA. ${ }^{2}$ Department of Applied Physical Sciences, University of North Carolina, Chapel Hill, NC 27599, USA. ${ }^{3}$ Department of Physics, North Carolina State University, Raleigh, NC 27695, USA. ${ }^{4}$ Nebraska Center for Materials and Nanoscience, University of Nebraska-Lincoln, Nebraska 68588, USA. ${ }^{5}$ Department of Chemistry, University of North Carolina, Chapel Hill, NC 27599, USA. These authors contributed equally: Yun Lin, Yanjun Fang, Jingjing Zhao, Yuchuan Shao. Correspondence and requests for materials should be addressed to J.H. (email: jhuang@unc.edu)
} 
O rganic-inorganic hybrid perovskites (OIHPs) have emerged as the most promising low-temperature solution-processable photovoltaic materials due to their intriguing properties like long carrier diffusion length, large absorption coefficient and low bulk trap density ${ }^{1-3}$. Despite the significant improvement in power conversion efficiency (PCE) achieved in the past few years ${ }^{4}$, the concern on the poor stability, especially the moisture stability, of OIHP materials remains a major hurdle for the commercialization of the OIHP solar cells ${ }^{5}$. The low stability stems mainly from the low formation energy and hydroscopic nature of the OIHP materials ${ }^{6}$. One approach to address the poor moisture stability of three-dimensional (3D) perovskite devices is to introduce layered OIHPs, or quasi-twodimensional (2D) perovskites, which contain sheets of $3 \mathrm{D}$ perovskites sandwiched by long organic ligands. The hydrophobic ligands can slow down the permeation of moisture into the lead iodide octahedron, and thus impede the decomposition of the OIHPs by moisture ${ }^{7,8}$. Since the photovoltaic devices fabricated by layered perovskite materials exhibit impressively better stability in some cases, these materials have recently attracted intensive attention, despite that their PCEs are still much lower than the $3 \mathrm{D}$ perovskite counterparts ${ }^{7-10}$

The further PCE improvement of layered perovskite solar cells relies on better understanding of their operation mechanism, which, however, remains largely elusive up to now. Tsai et al. ${ }^{9}$ reported that the layered perovskite film fabricated with hot-casting spincoating method showed the preferential alignment of the layered crystallographic planes along the out-of-plane direction, and assigned the high mobility in the crystallographic planes of the inorganic perovskite components is the main reason for the efficient charge carrier extraction and thus high PCE above $12 \%$ in layered perovskite solar cells. Nevertheless, the underlying working mechanism for solar cells involving layered perovskites is still not clear. For example, the morphology, phase compositions and distribution are not known yet for the perovskite thin films prepared with a composition for layered perovskites. Liu et al. ${ }^{11}$ observed very different photoluminescence (PL) properties from the top and bottom film surface, which was explained by different layered perovskite phases with increasing $n$ values naturally aligned along the vertical direction to the substrate. In addition, it is still an open question of how the photogenerated excitons efficiently dissociate in layered perovskites with exciton binding energy much larger than thermal energy at room temperature ${ }^{12-14}$. Blancon et al. ${ }^{15}$ proposed that the low energy edge states in layered perovskites can facilitate exciton dissociation. However, a full picture of exciton diffusion to edge states and free charge carrier extraction is still missing.

For a better understanding of the working principle of layered perovskite solar cells, in this study, we carefully examine the morphology of the hot-cast layered perovskite thin films at both microscopic and nanoscopic scales. The results show that layered perovskite thin films comprise multiple layered perovskite phases surrounded by $3 \mathrm{D}$-like perovskites. The layered flakes are much smaller than the thin film thickness. Based on the observed morphology, we propose a model for the operation of realistic layered perovskite solar cells which involves energy transfer from layered to $3 \mathrm{D}$-like perovskites and charge collection through the 3D-like perovskite network.

\section{Results}

Material preparation and device evaluation. Achieving efficient layered perovskite solar cells is essential to establish the material platform for investigating the working mechanism. In this study, layered perovskite films with a nominal composition of $(\mathrm{BA})_{2}(\mathrm{MA})_{3} \mathrm{~Pb}_{4} \mathrm{I}_{13} \quad\left(\mathrm{BA}=\mathrm{CH}_{3}\left(\mathrm{CH}_{2}\right)_{3} \mathrm{NH}_{3}{ }^{+} ; \mathrm{MA}=\mathrm{CH}_{3} \mathrm{NH}_{3}{ }^{+}\right)$ were fabricated by hot-casting method. The precursor solutions were prepared by dissolving specific stoichiometric quantities of n-butylammonium iodide (BAI), methylammonium iodide (MAI) and $\mathrm{PbI}_{2}$ in $\mathrm{N}, \mathrm{N}$-dimethylformamide (DMF). The X-ray diffraction (XRD) pattern of layered perovskite films is shown in Fig. 1a. Three diffraction peaks at $14.20^{\circ}, 28.48^{\circ}$ and $43.28^{\circ}$ were well resolved, which match the diffraction peak positions of $(\overline{1} 1 \overline{1})$, (202) and (313) crystallographic planes from layered perovskite $(\mathrm{BA})_{2}(\mathrm{MA})_{3} \mathrm{~Pb}_{4} \mathrm{I}_{13}$, respectively ${ }^{9}$. However, the diffraction peaks of (110), (220) and (314) crystallographic planes from 3D perovskite $\mathrm{MAPbI}_{3}$ located at $14.05^{\circ}, 28.45^{\circ}$ and $43.13^{\circ}$ or $(\overline{1} 1 \overline{1})$, (202) and (313) crystallographic planes from layered perovskite $(\mathrm{BA})_{2}(\mathrm{MA})_{n-1} \mathrm{~Pb}_{n} \mathrm{I}_{3 n+1}(n=2,3)$ also agreed with the XRD pattern quite well ${ }^{16-18}$. The calculated diffraction angle $2 \theta$ values of layered perovskites ( $n=2$ to 4 ) were summarized (Supplementary Table 1, Supplementary Note 2). The difference of $2 \theta$ values between the same crystallographic planes of $(\mathrm{BA})_{2}(\mathrm{MA})_{n-1} \mathrm{~Pb}_{n} \mathrm{I}_{3 n+1}(n=2$ to 4$)$ are within $0.12^{\circ}$, which are negligible compared to the FWHM (full width at half maximum) of the diffraction peaks (greater than $0.45^{\circ}$ for the XRD result presented here), making it even more difficult to index the different perovskite phases. There are not clear peaks at diffraction angles $(2 \theta)$ below $14^{\circ}$ which are characteristic of low-angle $\{010\}$ reflections in layered crystals ${ }^{18}$. In order to further determine the phase composition, XRD measurements of scraped powder of thin films were also conducted. Several extra diffraction peaks are present in powder XRD pattern (Supplementary Figure 1). After comparing it with the XRD patterns of $3 \mathrm{D}$ perovskite powders, we concluded that these high-angle diffraction peaks more likely originate from $3 \mathrm{D}$ perovskites, indicating the coexistence of layered and 3D-like perovskite phases in layered perovskite films. Weak diffraction peaks at low-angle (less than $14^{\circ}$ ) can be observed in the powder XRD spectrum; the absence of such peaks in layered perovskite thin film samples might be explained by the small amount of randomly oriented layered perovskite phases present. Exciton-like absorption peaks at $1.94 \mathrm{eV}(n=4)$ and $2.05 \mathrm{eV}(n=3)$ in Fig. $1 \mathrm{~b}$ indicate the films prepared from mixed precursor solution contain layered perovskites ${ }^{11}$. The blue-shift of absorption in the long wavelength region from $3 \mathrm{D}$-like perovskite phase may be ascribed to quantum confinement effect of small perovskite crystallites produced by bulking amine ligands effect, and similar results have been reported elsewhere ${ }^{19,20}$. As the scanning electron microscopy (SEM) image shown in Fig. 1c reveals, uniform, pinholefree and compact layered perovskite films were obtained by the hot-casting technique. The photovoltaic devices were fabricated with a structure of indium tin oxide (ITO)/poly(bis(4-phenyl) (2,4,6-trimethylphenyl)amine) (PTAA)/layered perovskites/[6,6]phenyl-C61-butyric acid methyl ester $(\mathrm{PCBM}) / \mathrm{C}_{60} / 2,9$-dimethyl4,7-diphenyl-1,10-phenanthroline (BCP)/Cu as shown in Fig. 1d. A reasonably high PCE of $12.7 \%$ was recorded from current density-voltage $(J-V)$ scanning, as shown in Fig. 1e, with an opencircuit voltage $\left(V_{\mathrm{OC}}\right)$ of $1.13 \mathrm{~V}$, short-circuit current density $\left(J_{\mathrm{SC}}\right)$ of $18.90 \mathrm{~mA} \mathrm{~cm}^{-2}$ and fill factor (FF) of $59 \%$. The external quantum efficiency (EQE) and integrated $J_{\mathrm{SC}}$ are shown in Fig. 1f. The integrated $J_{\mathrm{SC}}$ of $18.57 \mathrm{~mA} \mathrm{~cm}^{-2}$ agrees well with the measured $J_{\mathrm{SC}}$ from $J-V$ scanning. The layered perovskite thin films used for the morphology investigation in this study were fabricated with the same method as those in our high PCE layered perovskite solar cells.

We also synthesized layered perovskite single crystals for anisotropic mobility studies. The layered perovskite single crystals were synthesized following the liquid phase crystallization method (Fig. 2a, left panel). Typically, for layered $(\mathrm{BA})_{2}(\mathrm{MA})_{2} \mathrm{~Pb}_{3} \mathrm{I}_{10}$ single crystal with $n=3$, lead(II) iodide, $n$ butylamine and methylammonium iodide in appropriate molar ratios were dissolved in hydriodic acid solvent by heating. Subsequently, the hot solution was slowly cooled to room 

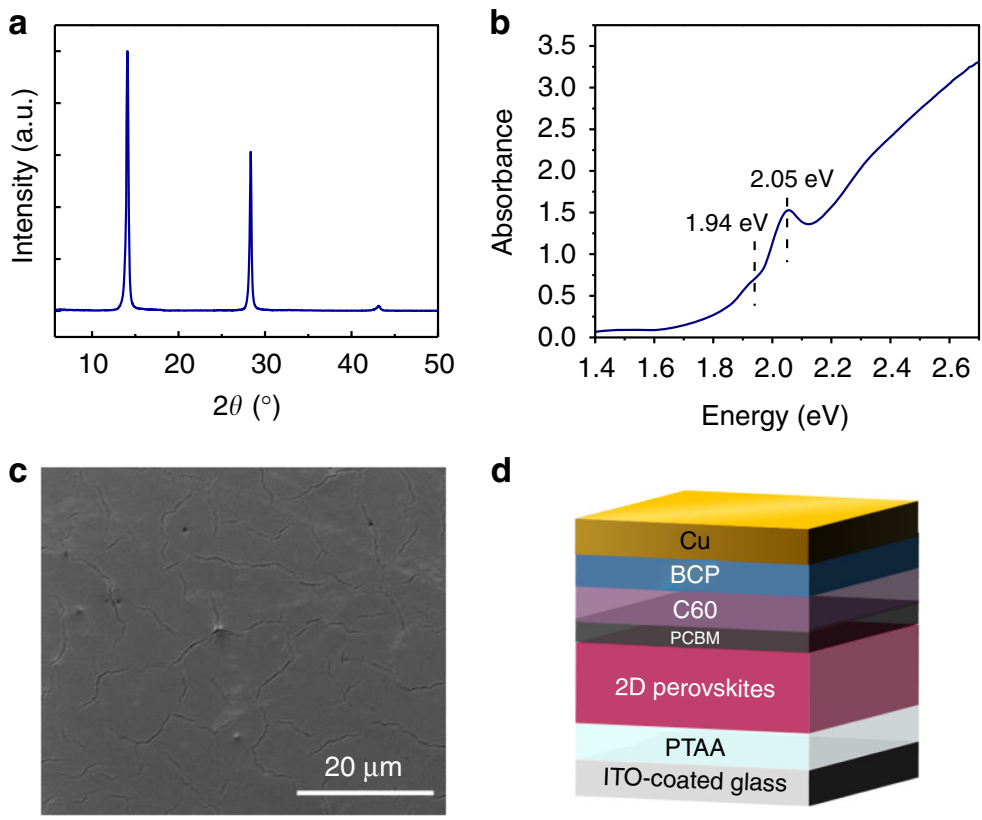

d
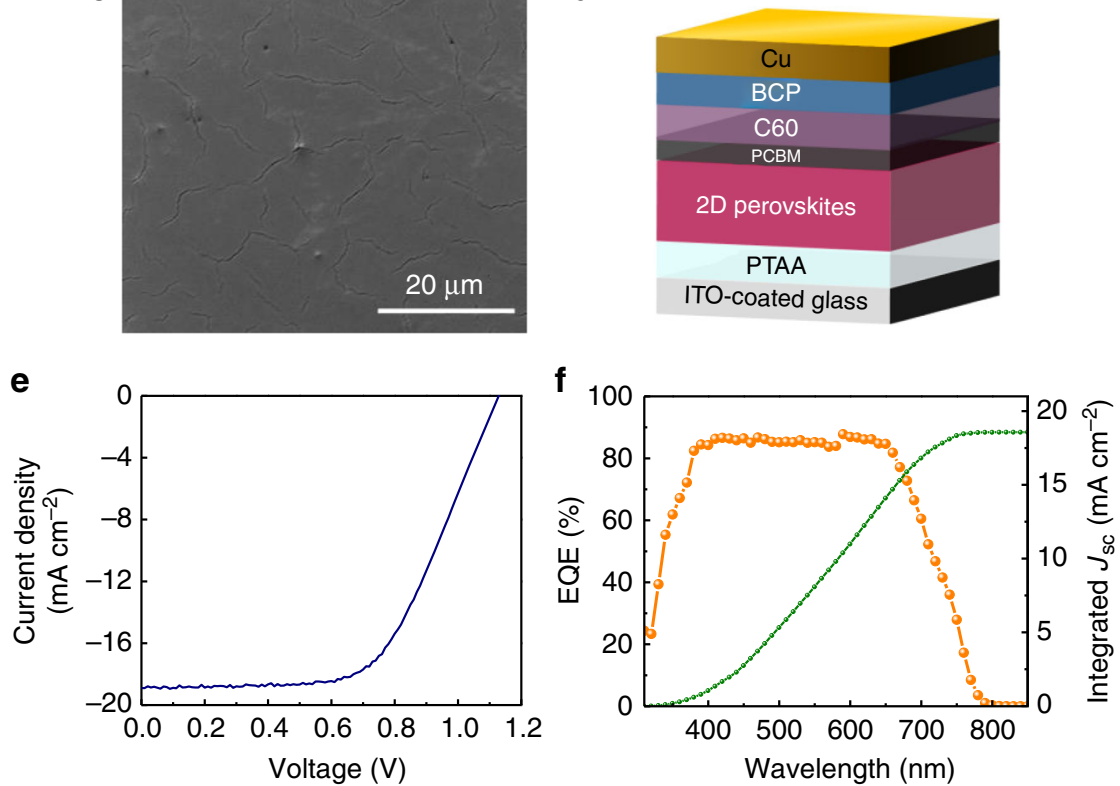

Fig. 1 Characterization of layered perovskite thin films and fabricated solar cells. X-ray diffraction (XRD) spectrum (a), absorption spectrum (b) and scanning electron microscopy (SEM) image (c) of layered perovskites films. $\mathbf{d}$ The layered perovskite photovoltaic device architecture. e Current densityvoltage $(J-V)$ curve of solar cells based on layered perovskites. $\mathbf{f}$ External quantum efficiency (EQE; orange) and integrated short-circuit current density $\left(J_{S C} ;\right.$ green $)$ as a function of wavelength for layered perovskite solar cells

temperature and layered perovskite $(\mathrm{BA})_{2}(\mathrm{MA})_{2} \mathrm{~Pb}_{3} \mathrm{I}_{10}$ single crystal platelets can be obtained at the solution-air interface (Fig. 2a, right panel). The XRD pattern (Fig. 2b) revealed three low-angle diffraction peaks between $2 \theta=5$ and $14^{\circ}$, which were indexed to be (040), (060) and (080) crystallographic planes of layered perovskite $(\mathrm{BA})_{2}(\mathrm{MA})_{2} \mathrm{~Pb}_{3} \mathrm{I}_{10}(n=3)$. The calculated $d$ spacing matches the distance between the discrete perovskite layers in $(\mathrm{BA})_{2}(\mathrm{MA})_{2} \mathrm{~Pb}_{3} \mathrm{I}_{10} \quad\{010\}$ reflection (Supplementary Table 2, also see Supplementary Note 2$)^{18}$. The optical absorption properties of the mechanically exfoliated layered perovskite single crystals $(n=3)$ are shown in Fig. 2c. The main exciton peak position, the onset of band-to-band absorption and the estimated exciton binding energy agree with previously reported results ${ }^{15}$.

Morphology of hot-cast layered perovskite thin films. The features of the absorption spectra of the hot-cast layered perovskite thin films indicate the presence of multiple phases of layered perovskites. The results from grazing-incidence wideangle X-ray scattering (GIWAXS) show further evidence of multiple phases and/or orientations (Supplementary Figure 2, Supplementary Figure 3 and Supplementary Note 1). As an exercise, the indexing of peaks from both layered perovskites $(n=4)$ and $3 \mathrm{D}$-like perovskite phases suggest that both layered and 3D-like perovskites could be present at certain orientations in the samples. Furthermore, as indicated by the few unindexed peaks, other phases/orientations are likely present as well besides these specific layered $(n=4)$ and $3 \mathrm{D}$-like phases. To obtain an indepth understanding of the morphology of the layered perovskite thin films, we investigated the distribution, size and orientation of the layered perovskite phases using a combination of nanoscopicand microscopic-level morphology characterizations.

Vertical phase segregation. First, the incident-angle-dependent photoluminescence was adopted to investigate the distribution of layered perovskite phases along the out-of-plane direction. Here, by changing the incident angle, we can control the penetration depth of the excitation laser into perovskite films (Fig. 3a). The PL measurement was conducted under two different excitation configurations, i.e., the incident laser beam with wavelength of $405 \mathrm{~nm}$ illuminated the perovskite films either from the air (front) side or the glass (back) side, as illustrated in the insets of Fig. 3 b and Fig. 3c, and the corresponding PL spectra are shown in Fig. 3b and Fig. 3c, respectively. For both front and back excitations, the PL spectra show several emission peaks, agreeing with the absorption spectra results and GIWAXS indications that the layered perovskite films comprise multiple perovskite phases and orientations. Five PL peaks at $576 \mathrm{~nm}, 615 \mathrm{~nm}, 650 \mathrm{~nm}, 673$ $\mathrm{nm}$ and $750 \mathrm{~nm}$ were observed. The PL peak at $750 \mathrm{~nm}$ can be assigned to $3 \mathrm{D}$-like perovskites ${ }^{15}$. The blue-shift of this peak compared to the PL peak of pure 3D perovskites which regularly 
a

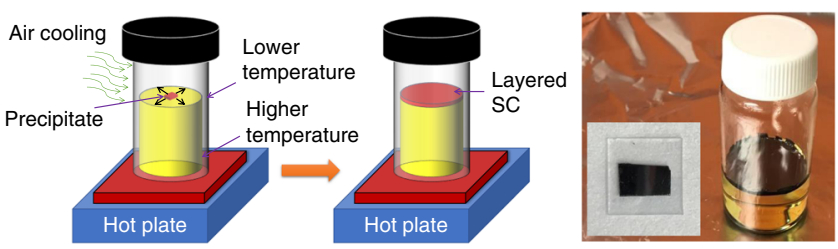

b
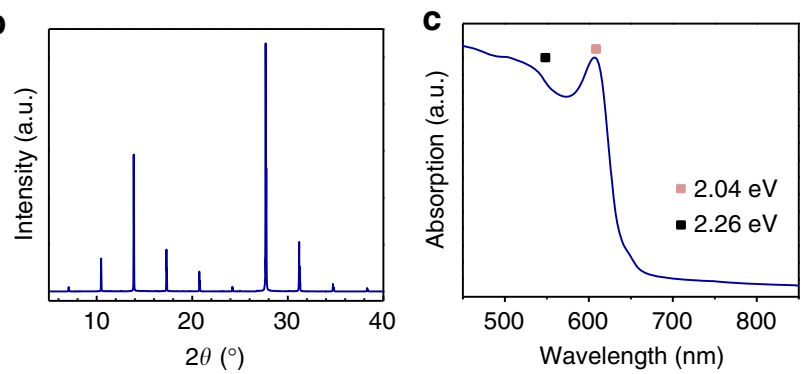

Fig. 2 Layered perovskite single crystals synthesis and characterization. a (left panel) Schematic of $\left.(B A)_{2}(M A)_{2} \mathrm{~Pb}_{3}\right|_{10}$ single crystal plate growth method; (right panel) photograph of $(B A)_{2}(\mathrm{MA})_{2} \mathrm{~Pb}_{3} \mathrm{l}_{10}$ single crystal platelet floating on the solution surfaces in a glass vial (the vial diameter is $25 \mathrm{~mm}$ ), and the hand-cut rectangular single crystal platelet stick to a glass substrate $(15 \mathrm{~mm} \times 15 \mathrm{~mm})$. X-ray diffraction (XRD) pattern (b) and absorption spectra $(\mathbf{c})$ of $\left.(B A)_{2}(M A)_{2} \mathrm{~Pb}_{3}\right|_{10}$ single crystal (the pink dot indicates the main exciton peak and the black dot indicates the position of the band-to-band absorption edge) locate at a position of $780 \mathrm{~nm}$ may also be ascribed to the quantum confinement effect. We observed that the film thickness has an obvious effect on this peak position, which shows a blueshift with the reducing film thickness, as shown in Supplementary Figure 4. After comparing these PL peaks with previous reports, the higher energy PL peaks at $576 \mathrm{~nm}, 615 \mathrm{~nm}, 650 \mathrm{~nm}$ and 673 $\mathrm{nm}$ can be assigned to emission from $(\mathrm{BA})_{2}(\mathrm{MA})_{n-1} \mathrm{~Pb}_{n} \mathrm{I}_{3 n+1}$ perovskites with $n=2,3,4$ and 5 , respectively ${ }^{11}$. The PL intensity had been normalized according to the intensity of the $750 \mathrm{~nm}$ emission peak.

The PL intensity ratio between different emission peaks obtained with different incident angles suggested that the distribution of different phases of layered perovskites was not uniform along the out-of-plane direction in the films. Under front excitation with incident light angle (the angle between the incident ray and the normal direction) of $89^{\circ}$, the incident laser at wavelength of $405 \mathrm{~nm}$ has the smallest penetration depth of 57.7 $\mathrm{nm}$ (the estimation of penetration depths was summarized in Supplementary Table 3). PL intensity of the dominant emission peak at $750 \mathrm{~nm}$ is 10 times stronger than those of 3 other emission peaks. With decreasing the incident angle and increasing penetration depth of excitation light, PL intensities from perovskite phases with $n=2,3$ and 4 gradually increase. Under back excitation with penetration of $64.9 \mathrm{~nm}$, PL intensities of the emission from perovskite phases with $n=2$ to 5 are comparable to that of perovskite phases with $n \approx \infty$. The difference of PL spectra between front excitation and back excitation and different excitation depths imply that the layered perovskite phases with smaller $n$ prefer to stay at the bottom, while the large $n$ layered or $3 \mathrm{D}$-like perovskite phases tend to stay a

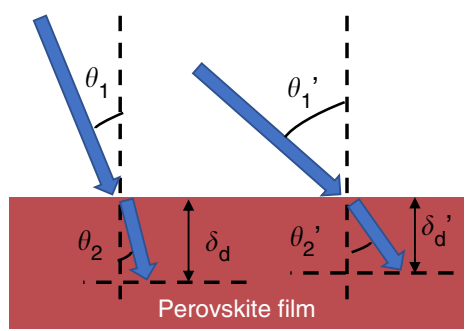

d

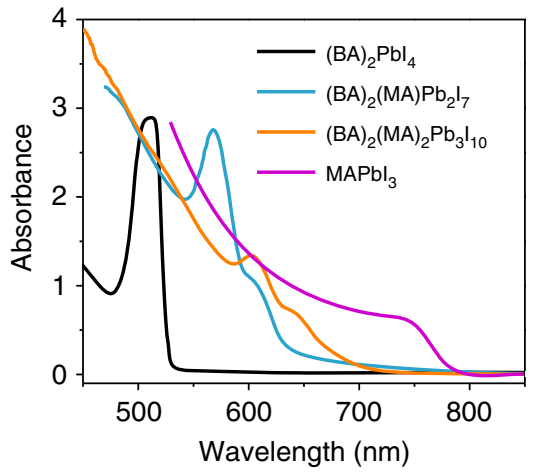

b

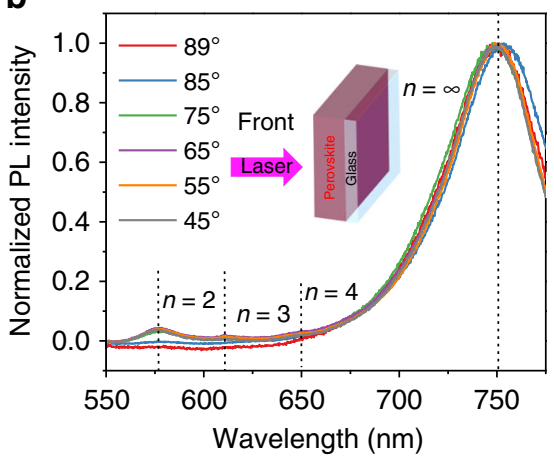

e

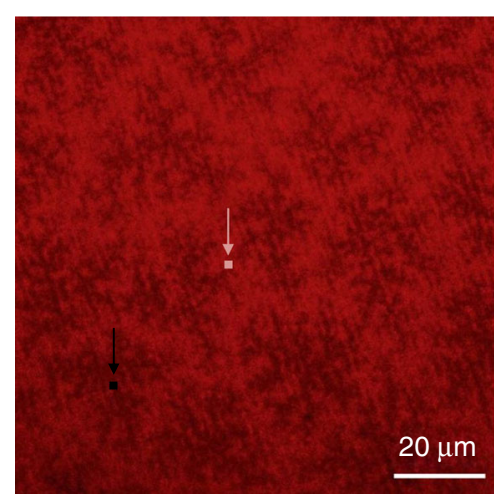

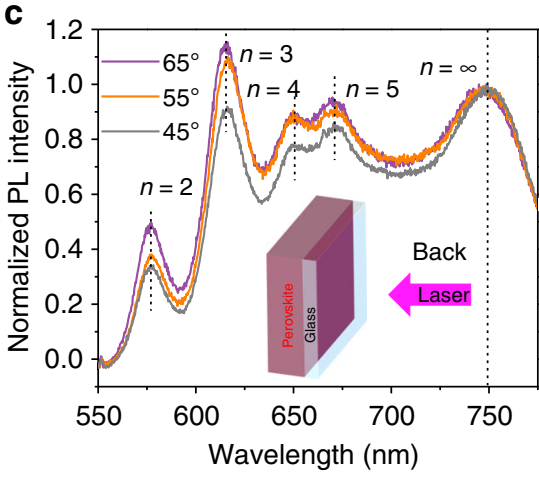

f

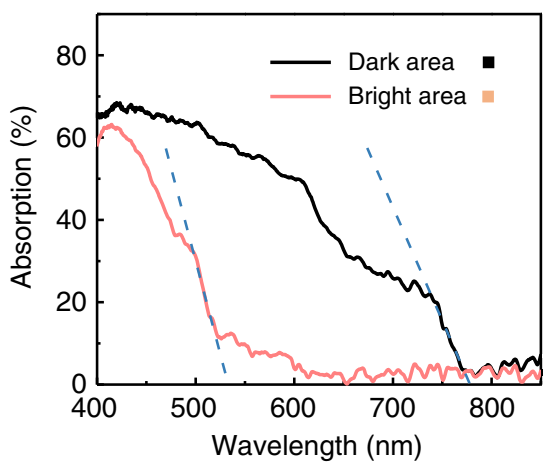

Fig. 3 Vertical and lateral phase segregation investigation. a Schematic of incident-angle dependent photoluminescence (PL) setup. b The incident-angledependent PL spectra of the layered perovskite films illuminated from the front sides (as illustrated in the inset) of the films. c The incident-angledependent PL spectra of the layered perovskite films illuminated from the back sides (as illustrated in the inset) of the films. $\mathbf{d}$ Absorption spectra of threedimensional (3D) $\mathrm{MAPbl}_{3}$ thin films and the layered $(B A)_{2}(\mathrm{MA})_{n-1} \mathrm{~Pb}_{n} \mathrm{I}_{3 n+1}$ perovskite thin films $(n=1$ to 3). e Optical mapping of the layered perovskite $(\mathrm{BA})_{2} \mathrm{MA}_{3} \mathrm{~Pb}_{4} \mathrm{l}_{13}$ films using an optical microscope in transmission mode. Typical bright and dark area are labeled with pink and black squares, respectively. f Absorption spectra measured in the bright and dark area labeled in e. The size of the measured area is $0.6 \times 0.6 \mu \mathrm{m}^{2}$, smaller than the labeled area 

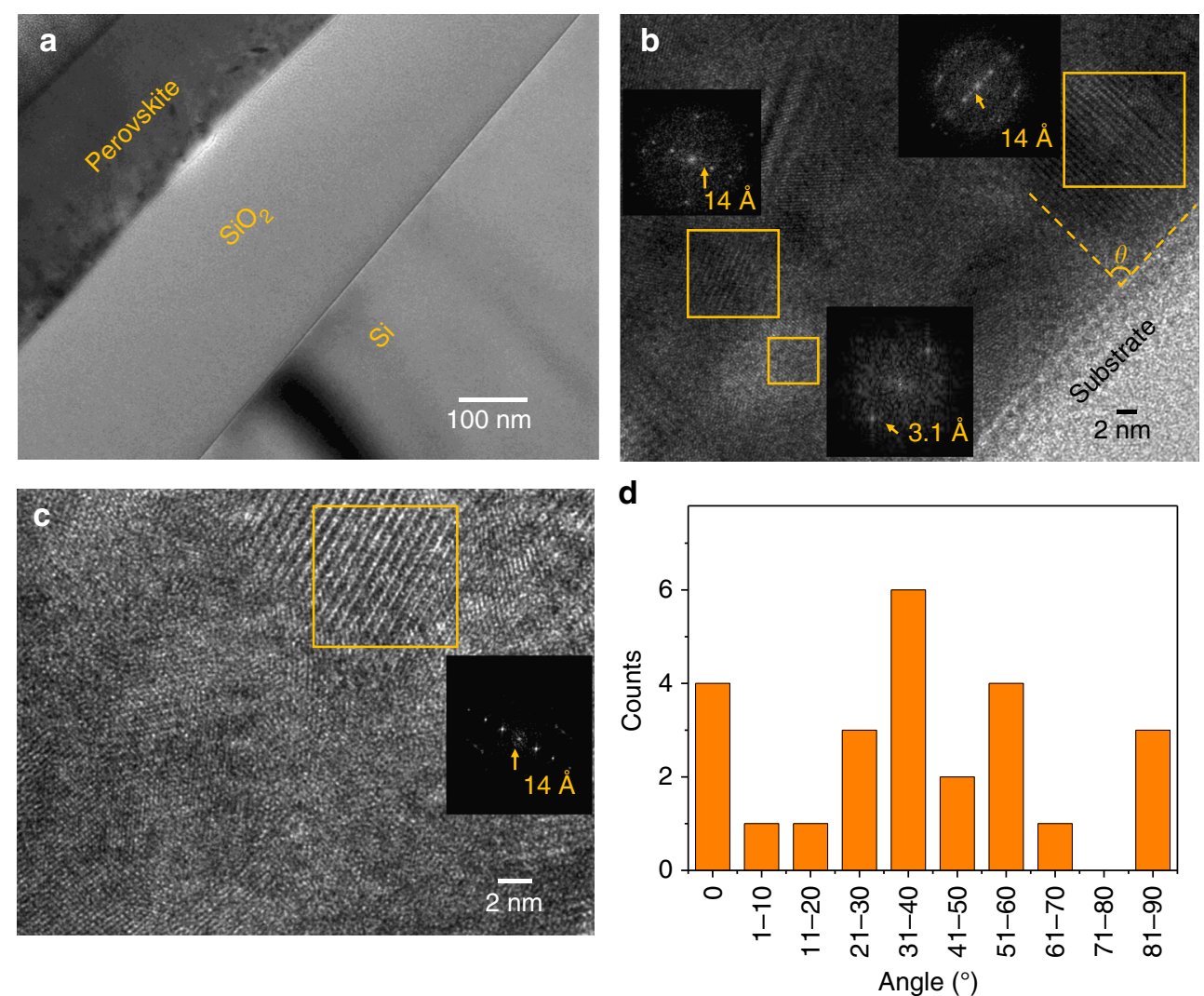

Fig. 4 High-resolution transmission electron microscope (HRTEM) images of cross-section of the spin-coated $B A_{2} M_{3} A_{3} P_{4} l_{13}$ thin films. a-c TEM images of the cross-section of the thin film at different locations and different magnifications. Inset: images of the fast-Fourier transforms (FFT) of the area in yellow square. $\mathbf{d}$ Statistical distribution of the angle $\theta$ between substrate and layered perovskites

close to the surface of the films. This is consistent with the previous study by Liu et al. ${ }^{11}$. The film thickness could have an effect on such graded distribution along vertical direction reported in previous work, and thicker films exhibit more dramatic difference in phase distribution throughout the sample thickness (as shown in Supplementary Figure 4) ${ }^{21}$.

Lateral phase segregation. After identifying the nonuniform distributions of layered perovskite and 3D-like perovskite phases in vertical direction, we conducted the optical transmission mapping measurement to study their lateral distributions by leveraging the differences between the absorption spectra of layered and 3D perovskites. As shown in Fig. 3d, 3D perovskite has much stronger absorption than layered perovskites in the wavelength range between $680 \mathrm{~nm}$ and $770 \mathrm{~nm}$. In the transmission mode, the incident light with wavelength less than $650 \mathrm{~nm}$ will be filtered after applying a $650 \mathrm{~nm}$ long pass filter. Therefore, the area with more layered perovskite phases appear brighter as less incoming light can be absorbed. In contrast, the area with more $3 \mathrm{D}$ perovskite phase appears darker in the transmission mapping images. For the hot-cast layered perovskite thin films, dendrite structure with the size in the range of several to tens of micrometers were observed, indicating the nonuniform distribution of different perovskite phases in the lateral direction (Fig. 3e). By further comparing the absorption spectra of the dark and bright area (Fig. 3f), we conclude that the dark areas correspond to $3 \mathrm{D}$-like perovskite phase while the bright areas correspond to layered perovskite phases, and layered perovskites was surrounded by $3 \mathrm{D}$-like perovskites.
Morphology at the atomic scale. After identifying the nonuniform distribution of multiple layered perovskite phases in both vertical and lateral directions, we further studied their size and orientation with high-resolution transmission electron microscope (HRTEM). The HRTEM images, as well as associated fastFourier transforms (FFT), of the thin film's cross-section shown in Fig. 4, convey a better picture of the overall morphology of the real layered films. In Fig. $4 \mathrm{~b}$, the $14 \AA$ lattice spacing corresponds to the $\mathrm{BA}_{2} \mathrm{PbI}_{4}(n=1)$ phase. Most of these layered domains have sizes in the range of several to tens of nanometers, much smaller than the film thickness. Surrounding the $\mathrm{BA}_{2} \mathrm{PbI}_{4}(n=1)$, the narrower plane spacing of $3.1 \AA$ indicates the presence of $3 \mathrm{D}$ $\mathrm{MAPbI}_{3}$. HRTEM allows us direct observation of the orientation of the layered perovskites. Very careful sample preparation utilizing the focused ion beam lift-out procedure is required to obtain a clear HRTEM image. Figure 4 b, c shows two typical HRTEM cross-sectional images at locations close to and away from the substrates. To reveal the orientation distribution of the layered perovskite phases, we randomly chose 25 areas of layered perovskite phases for HRTEM study. The results are shown in Fig. $4 \mathrm{~d}$. Here, $\theta$ is the angle between the substrate and (001) planes of layered perovskites. Thus, $90^{\circ}$ and $0^{\circ}$ represent that the layered perovskite layer is perpendicular to and parallel with the substrate, respectively. From Fig. 4d, there might be apparent preferred orientation of the layered perovskite regions, but one would need more HRTEM images for better statistics.

Model of growth kinetics and device operation mechanism. A model of film formation mechanism is proposed to explain the observed morphology, i.e., the presence of both vertical and lateral phase segregation, and with most of layered domains with sizes in the range of several to tens of nanometers are surrounded 
a

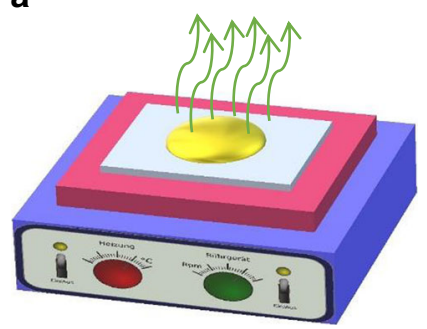

b

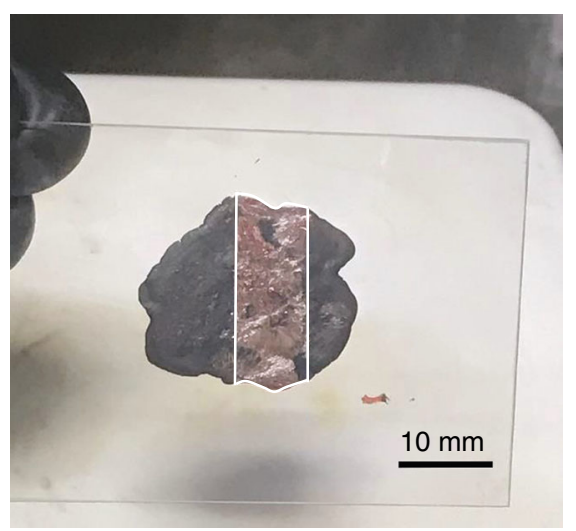

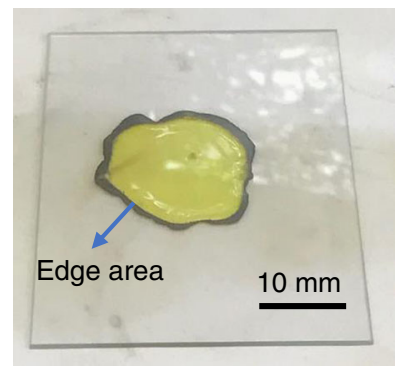

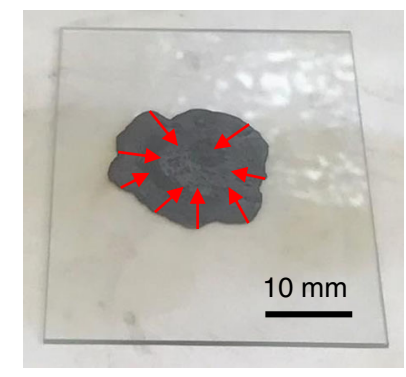

C

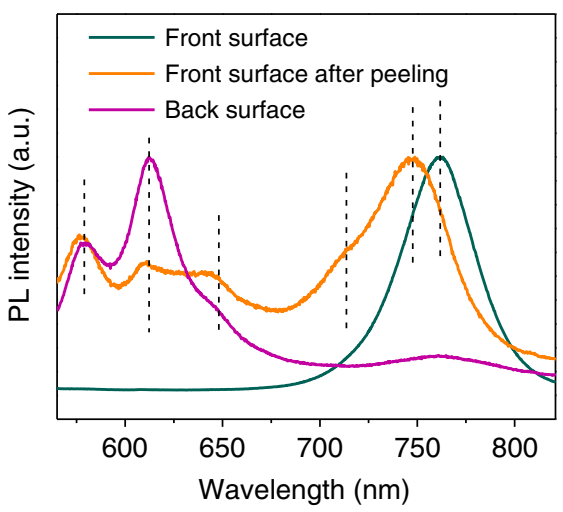

Fig. 5 Optical images and photoluminescence (PL) spectra of top-crust peeling-off test. a Schematics of top-crust peeling-off test setup. A glass substrate $\left(40 \mathrm{~mm} \times 50 \mathrm{~mm}\right.$ ) with $100 \mu \mathrm{L} \mathrm{BA} \mathrm{MA}_{3} \mathrm{~Pb}_{4} \mathrm{I}_{13}$ precursor solution ( $2 \mathrm{M}$ in $\mathrm{N}, \mathrm{N}$-dimethylformamide (DMF)) on top was placed on a hot plate at $70{ }^{\circ} \mathrm{C}$. The yellow solution begun to turn dark in the edge, and this dark area gradually spread into the center as indicated by the red arrows, forming a top-crust. $\mathbf{b} A$ Kapton tape was used to partially peel off the top-crust, and the revealed yellow precursor solution underneath that has not yet crystallized started to turn red under heating. After the solution had dried, the glass substrate was removed from the hot plate. The area inside the white frame represented the crystallized front surface revealed after the top-crust was removed by Kapton tape. c PL spectrum of different areas in $\mathbf{b}$

by $3 \mathrm{D}$-like perovskites. In the hot-casting process, hot precursor solution was casted on a hot spinning substrate. It is highly likely that the precursor solution at the liquid-air interface would reach supersaturation first due to the fast evaporation of DMF solvent at the surface, which has been confirmed by a top-crust scraping test $^{22}$. As shown in Fig. 5a, b and Supplementary Movie 1, we find that 3D-like crystal tends to first precipitate prior to layered perovskites. This is a similar top-crust peeling-off test but using precursor material for layered perovskites of $n=4$. The fact that black shell first formed proves that $3 \mathrm{D}$-like crystals are more easily to form, otherwise the shell would be dark-red colored for $n=4$ layered perovskites. As shown in Fig. 5c, a PL peak at 762 $\mathrm{nm}$ was probed for the formed shell, which can be assigned to 3D-like perovskites. The consumption of much less BA in top 3D-like perovskite layer actually pushes more BA toward the substrate side so that the ratio of BA to MA gradually increased in the leftover solution. The more BA-rich solution accelerates the layered perovskite nucleus formation and allows the formation of layered perovskites, though there is still a competition of 3D-like perovskites and layered perovskites. Actually PL emissions from bottom layer after peeling off the top-crust or excite from glass substrate side show the presence of layered perovskites with $n=2$ to 4 , a group of layered perovskites with much large $n$ values and 3D-like $(n \rightarrow \infty)$ perovskites in the film crystallized from the leftover precursor solution underneath the top-crust.

This basically explains the observed vertical phase separation with more layered perovskite with smaller $n$ towards the substrate site, and lateral phase separation in both micrometer and nanometer scales. This size of layered perovskite grains is limited by how fast the BA ligands can diffuse to the site for layered perovskite growth, and the competition of 3D-like and layered perovskites may interrupt the growth of large grain layered perovskites, as evidenced by the HRTEM images. The competition of 3D-like and layered perovskite formation also indicates that the film morphology should be very sensitive to the process condition such as temperature or solvent drying speed. This is again supported by the study of layered perovskite formation by slowing down the 3D perovskite formation by adding dimethyl sulfoxide (DMSO) or $N$-methyl-2-pyrrolidone (NMP) to the precursor solution. DMSO in precursor solution has been shown to promote the layered perovskite growth ${ }^{23}$, which is also observed here by the much strong PL emission from the layered perovskites in both front and back side of the films, and clear XRD peaks at low diffraction angle (Supplementary Figure 5, Supplementary Note 3). This basically can be explained by the sloweddown $3 \mathrm{D}$ perovskite formation by DMSO or NMP due to the formation of intermediate phases. Sargent and colleagues ${ }^{21}$ have reported the observation of intermediate phase in reduceddimensional metal halide perovskites when DMSO/NMP is used in solutions. This might also arise from differences in the solubility of the cation (BA or MA) in the different solvents.

Based on the optical, XRD and HRTEM results discussed above, the growth kinetics and morphology of layered perovskite thin films fabricated by hot-casting is summarized in Fig. 6. The $3 \mathrm{D}$-like crystal tends to precipitate first. As more and more 3Dlike crystals precipitated, the ratio of BA to MA in leftover solution gradually increased and the BA-rich solution facilitated the afterward layered perovskite nucleus formation. As layered perovskite grains grew up, the BA/MA ratio in the surrounding solution gradually decreased and such MA-rich solution will again facilitate the 3D-like perovskite nucleation and growth, forming the morphology that layered perovskite grains with size of several to tens of $\mathrm{nm}$ were embedded in 3D-like perovskite 

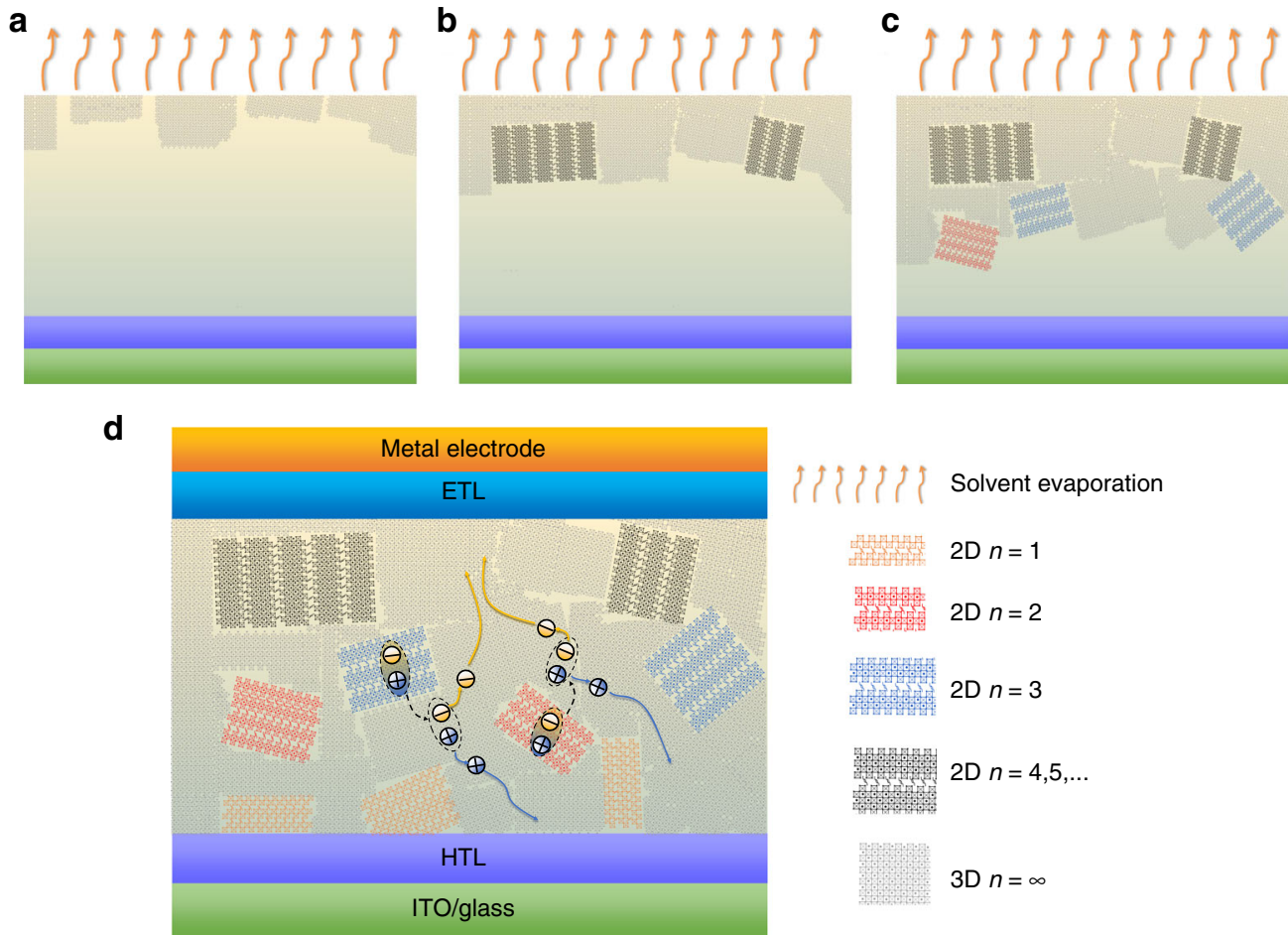

Fig. 6 Growth kinetics, morphology and device operation mechanism model. a-c The growth kinetics and morphology model. d The device structure is indium tin oxide (ITO)/hole transport layer ( $\mathrm{HTL}$ )/layered perovskite film/electron transport layer (ETL)/metal electrode

network which agrees quite well with the atomic-scale morphology that we observed in HRTEM results. The spatial distribution of these layered perovskites was nonuniform in both vertical and lateral directions, and more layered perovskite phases with small $n$ tend to segregate close to the substrate.

From the morphology scenario directly observed, we proposed a model for the operation of perovskite solar cells with layered perovskites (Fig. 6d): To contribute to the PCE of layered perovskite solar cells, the excitons generated in layered perovskite phases need to go through the layered/3D-like perovskite interface via a charge transfer or energy transfer. The transient absorption measurements were performed with the layered perovskite thin films $(n=4)$. The samples were excited from the back side (glass side) by a $20 \mu \mathrm{J} \mathrm{cm}^{-2} 570 \mathrm{~nm}$ pump beam. As shown in Supplementary Figure 6, the TA signals with a negative sign around $572 \mathrm{~nm}, 604 \mathrm{~nm}, 639 \mathrm{~nm}$ and $704 \mathrm{~nm}$ are attributed to the resonance of $n=2,3,4$, and 3D-like phase, respectively. For 3D-like and high $n$ number 2D $(n>3)$ phases, the red-shift of the peak wavelength is caused by hot carrier cooling as the excitation energy is far above their bandgap. Growth of the 3Dlike perovskite bleach peak and fast decay dynamics at $572 \mathrm{~nm}$ and $604 \mathrm{~nm}$ suggests an energy/charge transfer process between small $n$ number 2D phase and 3D-like perovskite phase. According to previous studies 24,25 , we speculate that the energy transfer is more dominant in this system, because the transfer process happens at a very early delay time scale (far before 500 ps). We speculate energy transfer from the larger bandgap layered perovskites to lower bandgap 3D-like perovskites across the ligands. The layered flakes observed in our study were generally few layers, and thus we expect most of energy may be transferred to 3D-like perovskites. After energy transfer, excitons are generated in 3D-like perovskites, which will dissociate into free charge quickly due to the very small exciton binding energy in 3D-like perovskites. Thus, the transport and collection of free charge carriers was dominated by 3D-like perovskite phase which form a percolation network in layered perovskite solar cells.
Carrier transport in layered perovskite solar cells. One way to verify whether photogenerated charges go through $3 \mathrm{D}$-like perovskite phase to be collected is to measure the charge extraction time needed for both the 3D and layered perovskites with a thickness of the devices. In view of the layered structure of layered perovskites and the insulating nature of the long chain organic cations, it is intuitively assumed by us that there would be large conductivity anisotropy along the in-plane and out-of-plane directions. However, there is no study yet to find out whether the long organic ligands really inhibit the out-of-plane carrier transport properties. Here we used $(\mathrm{BA})_{2}(\mathrm{MA})_{2} \mathrm{~Pb}_{3} \mathrm{I}_{10} \quad(n=3)$ single crystals (SCs) as a model system to measure the carrier mobility along these two directions with the space charge limited current (SCLC) method. Hole-only and electron-only devices were fabricated by sandwiching the layered perovskite SC between $\mathrm{Au}$ electrodes or $\mathrm{C}_{60}$ /bathocuproine $(\mathrm{BCP}) / \mathrm{Cu}$ electrodes, respectively, because of the large electron (hole) injection barrier at the layered perovskite/ $\mathrm{Au}\left(\mathrm{C}_{60} / \mathrm{BCP} / \mathrm{Cu}\right)$ interface. For the out-of-plane mobility measurement, a gap larger than the crystal thickness is intentionally left between the electrode edge and the crystal edge when depositing the electrodes on the crystals, to avoid the influence of possible edge conductance of the crystal, as schematically shown in the inset of Fig. 7a. The device dark current density $(J)$ as a function of bias $(V)$ of the vertical structured hole-only device is shown in Fig. 7a, which can be divided into Ohmic response region $(J \propto V)$ at the low bias, and the trap-filled SCLC region $\left(J \propto V^{2}\right)$ under large bias. The $J-V$ curve at the SCLC region can be well-fitted by the Mott-Gurney law:

$$
J=\frac{9 \varepsilon \varepsilon_{0} \mu V^{2}}{8 L^{3}}
$$

where $\varepsilon$ is the relative dielectric constant that equals to 5 for $(\mathrm{BA})_{2}(\mathrm{MA})_{2} \mathrm{~Pb}_{3} \mathrm{I}_{10}, \varepsilon_{0}$ is the vacuum permittivity, $\mu$ is the carrier mobility and $L$ is the distance between the electrodes. The hole 

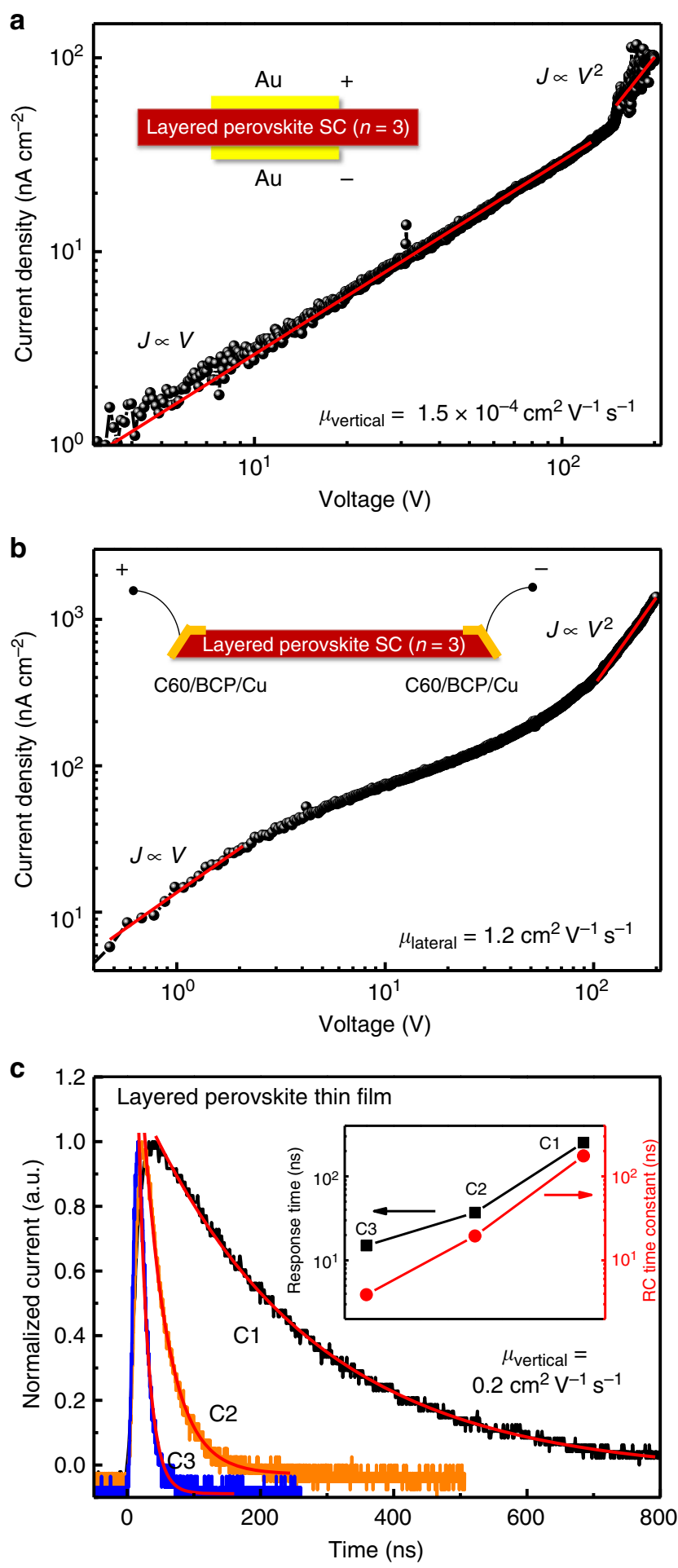

Fig. 7 Carrier mobility study in layered perovskite single crystals and thin films. Current density-voltage curves for a hole-only vertical structured layered perovskite single crystal (SC) device (a) and an electron-only lateral-structured layered perovskite SC device (b). The insets show the device structure of vertical and lateral devices, respectively. c Transient photocurrent (TPC) curves of layered perovskite thin film solar cells with decreased working area (from $\mathrm{C} 1$ to $\mathrm{C} 3$ ). The inset is the measured RC time constant of the device as well as the fitted decay time of the TPC curves with different device working areas. The red lines are the fitting curves to the data mobility along the out-of-plane direction is fitted to be $1.5 \times 10^{-4}$ $\mathrm{cm}^{2} \mathrm{~V}^{-1} \mathrm{~s}^{-1}$, which is comparable to carrier mobilities in organic semiconductors ${ }^{26-28}$. The in-plane electron mobility derived from the $J$ - $V$ curve of the lateral-structured electron-only device is approximately $1.2 \mathrm{~cm}^{2} \mathrm{~V}^{-1} \mathrm{~s}^{-1}$, which is nearly four orders of magnitude larger than that along the out-of-plane direction, and is smaller but close to that of $3 \mathrm{D}$ perovskites (Fig. $7 \mathrm{~b}$ ). For the electron-only vertically structured device, however, the $J-V$ curve was dominated by the Ohmic region all the way up to the maximum bias applied, which made it difficult to derive the electron mobility along this direction. Nevertheless, the comparable conductivity at the Ohmic region for the hole-only and electron-only devices (Supplementary Figure 7) indicates that the electron mobility and hole mobility of $(\mathrm{BA})_{2}(\mathrm{MA})_{2} \mathrm{~Pb}_{3} \mathrm{I}_{10}$ along the out-of-plane direction should be within the same order of magnitude.

The knowledge of the anisotropic mobility in layered perovskite crystals enabled us to determine the main charge transport path by measuring the carrier transit time in the layered perovskite thin films. Here, we used the transient photocurrent (TPC) method to measure the carrier transit time of the BA-based layered perovskite thin film solar cells (with precursor composition of $n=4)$ with a device structure of ITO/PTAA/layered perovskite (550 nm thick)/PCBM/C60/BCP/Cu, as shown in Fig. 7c. For the TPC measurement, the RC constant of the device was carefully controlled to be smaller than the carrier transit time by reducing device area. The derived low limit for the mobility of the layered perovskite films from TPC was $0.2 \mathrm{~cm}^{2} \mathrm{~V}^{-1} \mathrm{~s}^{-1}$ by neglecting the transit time in other buffer layers. This mobility is comparable to the in-plane carrier mobility of layered perovskite SC, or measured mobility of polycrystalline 3D perovskite materials ${ }^{3,29}$, but much larger than that of layered perovskites along the out-ofplane direction. Since we do not see layered flakes are large enough to connect electron and hole transport layers, the results agree well with our scenario that the charge extraction process in the layered perovskite thin films is dominated by the $3 \mathrm{D}$-like perovskite phase.

\section{Discussion}

The morphology observed in this study can help the understanding of the device efficiency limitation and stability enhancement in layered perovskite solar cells. The layered/3Dlike mixture still allows the charge extraction out of the device through the 3D-like network if the film thickness is not too large. This generally explains the high EQE of 80 to $90 \%$ achieved in the wavelength range of 400 to $650 \mathrm{~nm}$. However, compared to the EQE spectrum of pure $3 \mathrm{D}$ perovskite solar cells, it is found that EQE within the wavelength range of 650 to $750 \mathrm{~nm}$ is much lower for the layered perovskite solar cells. This may be caused by the inefficient absorption of the incident photons at this wavelength range, or due to the less efficient energy transfer from the layered phases with larger $n$ values to the 3D-like phase because of either the larger flakes or the smaller bandgap difference between them. This presents a challenge to efficiently harvesting the incident photon energies for the layered perovskite solar cells, which is the main reason that the $J_{\mathrm{SC}}$ of the layered perovskite solar cells is lower than that of their 3D counterparts. Increasing the thickness of such films to enhance absorption generally does not always result in enhanced $J_{\mathrm{SC}}$, may be due to the fact that the layered perovskite flakes with non-ideal orientation embedded in 3D-like perovskite would still impede the charge transport and thus cause charge recombination. The $V_{\mathrm{OC}}$ of layered perovskite solar cells should be limited by the smallest bandgap phase when the photoactive layer is a mixture of multiple phases with different band gaps. This is the reason that the obtained $V_{\mathrm{OC}}$ of $1.13 \mathrm{~V}$ is 
comparable to the value widely achieved in 3D perovskite solar cells despite the layered perovskites has larger bandgap than their 3D counterpart. In terms of fill factor, it is much lower for the layered perovskite solar cells in comparison to the $3 \mathrm{D}$ counterparts, which may be caused by the increasing charge carrier recombination occurs at the layered/3D-like perovskite interface.

Regarding the better environmental stability of layered perovskite solar cells observed in some cases, it is generally believed that this is mainly benefited from the protection of the organic ligand of the layered perovskite materials. However, the real morphology result shown here reveals that the layered perovskite films formed by hot-casting method is mainly composed of 3Dlike perovskite phases with more 3D-like phases preferentially distributed on the top surface. It is intriguing to understand why their long-term stability can outperform the 3D counterparts in some cases. We speculate the enhanced stability of perovskite solar cells with addition of some $2 \mathrm{D}$ additive may be contributed by multiple factors. First of all, the organic ligands added in the precursor solution for layered perovskite formation must stay somewhere if they do not convert all the 3D perovskites into layered perovskites. They most likely will stay at grain boundaries which may contribute to the formation of more robust grain boundaries. Our recent studies showed that defective grain boundaries may initiate and facilitate the film degradation, because moisture, oxygen or constituting ions in perovskites can migrate much faster along them ${ }^{30}$. As a matter of fact, our previous study on the ion migration property of the layered perovskite films fabricated by the same method as reported here showed that the ion migration was largely suppressed up to $330 \mathrm{~K}$ in layered perovskite films both in dark and under illumination 31,32 . This can significantly alleviate the ion migration-induced degradation of the perovskite layer, especially under illumination. Second, the addition of ligands for layered perovskites may also relieve the strain in the formed films and thus enhance the intrinsic stability of the films. The presence of lattice strain was recently observed by us in perovskite films formed through annealing process, and the lattice strain accelerates the degradation of perovskite films because ion migration in perovskite films is accelerated by the presence of $\operatorname{strain}^{33}$. The relief of strain can suppress the ion migration process and thus improve the photo-stability of $\mathrm{MAPbI}_{3}$ thin films. We propose that the better stability of layered perovskite solar cells may also have contribution from the less-strained perovskite layer in comparison to the pure 3D perovskite films, which is confirmed by the very small XRD peak shift between the layered perovskite film on substrate and the freestanding strain-free perovskite powders scratched from the substrate (see Supplementary Figure 8).

In summary, we conduct nanoscopic- and microscopic-level morphology characterization to reveal the real morphology of the hot-cast layered perovskite solar cells. Based on that, we propose a model to explain their working mechanism: the photons absorbed by the layered perovskite phases can contribute to the photocurrent by energy transfer process from the larger bandgap layered phases to the smaller bandgap 3D-like phase, and the carrier transport within the perovskite layer is mainly through the 3D-like phase which forms a percolating network across the vertical direction of the solar cell devices.

\footnotetext{
Methods

Preparation of perovskite solar cells. The ITO-coated glass substrates were cleaned sequentially in acetone and isopropyl alcohol under sonication for $30 \mathrm{~min}$, twice for each step. After drying in a vacuum oven, the substrates were treated by ultraviolet (UV) ozone for $15 \mathrm{~min}$ and then transferred to nitrogen glovebox for use. The precursor solutions were prepared by dissolving specific stoichiometric quantities of BAI, MAI and $\mathrm{PbI}_{2}$ in DMF (molar ratio 2:3:4 for $n=4$ ) with a concentration of $1.0 \mathrm{M}$. PTAA layers as the hole transport layer were firstly
}

deposited on ITO substrates by spin-coating PTAA solution ( $0.2 \mathrm{wt}$.\% in Toluene) at $6000 \mathrm{rpm}$ for $35 \mathrm{~s}$, followed by thermal annealing at $100^{\circ} \mathrm{C}$ for $10 \mathrm{~min}$. Then, the pre-heated PTAA-coated substrate was immediately transferred to the vacuum chunk of spin coater within $5 \mathrm{~s}$, followed by dropping the hot perovskite precursor solution $\left(60 \mu \mathrm{L}, 70{ }^{\circ} \mathrm{C}\right)$ on hot substrate and spin-coating at $5000 \mathrm{rpm}$ for $20 \mathrm{~s}$ without ramp. The hot-cast films were then annealed at $85^{\circ} \mathrm{C}$ for $3 \mathrm{~s}$. PCBM as the electron transport layers were directly deposited on top of perovskite layers by spin-coating the solution ( $20 \mathrm{mg} \mathrm{mL}^{-1}$ in 1,2-dichlorobenzene (DCB)) at $6000 \mathrm{rpm}$ for $35 \mathrm{~s}$ and afterwards annealed at $100^{\circ} \mathrm{C}$ for $30 \mathrm{~min}$. C60 (buffer layers) and BCP (hole blocking layers) were thermally evaporated sequentially, which is followed by the thermal evaporation of $\mathrm{Cu}$ electrode.

Film characterization. The XRD experiments were performed by a Bruker D8 Discover Diffractometer utilizing $\mathrm{Cu}$ Ka radiation. The SEM images were obtained with a Quanta 200 FEG ESEM scanning electron microscope. The absorption spectra were recorded by an Evolution 201/220 UV/vis (visible) Spectrophotometer. Photoluminescence measurements were performed with a Horiba 320 detector, and all the PL measurements were conducted in reflection mode. The transmission mapping of layered $(\mathrm{BA})_{2} \mathrm{MA}_{3} \mathrm{~Pb}_{4} \mathrm{I}_{13}$ thin films was taken by an optical microscope Olympus BX61 combined with a $650-\mathrm{nm}$ long pass filter.

Photovoltaic device characterization. The photocurrent density-voltage curves of the photovoltaic devices were recorded by a Keithley 2400 Source-Meter with homemade testing software. The devices were exposed to a xenon-lamp based solar simulator (Oriel 67005, $150 \mathrm{~W}$ ) under AM $1.5 \mathrm{G}$ irradiation $\left(100 \mathrm{~mW} \mathrm{~cm}^{-2}\right)$, the light intensity was calibrated by a Si photodiode (Hamamatsu S1133). The J-V tests were swept along forward direction with scan rate of $0.1 \mathrm{~V} \mathrm{~s}^{-1}$ and delay time of 100 ms. The EQE measurement was conducted using a Newport QE measurement kit, and the intensity of monochromatic beam of light was calibrated with a reference silicon photodiode and focused onto the working areas of devices.

Synthesis of the layered perovskite single crystals. For layered perovskite single crystal with $n=3$ synthesis, the liquid phase crystallization method was used as reported previously ${ }^{11,34,35}$. The $1.844 \mathrm{~g}$ lead(II) iodide, $198 \mu \mathrm{L} n$-butylamine and $0.477 \mathrm{~g}$ methylammonium iodide were completely dissolved in $6 \mathrm{~mL}$ hydriodic acid at $110^{\circ} \mathrm{C}$. Subsequently, the hot solution was slowly cooled down to room temperature, typically decreasing $10^{\circ} \mathrm{C}$ for $1 \mathrm{~h}$. Layered perovskite single crystal plate will gradually precipitate and grow at the solution-air interface.

Characterization of the layered single crystal. The successful synthesis of layered perovskite single crystal with $n=3$ was confirmed by the UV/vis absorption and XRD measurement. The Absorption spectra were recorded using an Evolution $201 \mathrm{UV} /$ Visible Spectrophotometer. The X-ray diffraction patterns were obtained by a Rigaku D/Max-B X-ray diffractometer in the Bragg-Brentano parafocusing geometry. A conventional copper target X-ray tube equipped in the diffracted-beam monochromator was set to $45 \mathrm{kV}$ and $40 \mathrm{~mA}$.

Grazing-incidence wide-angle x-ray scattering experiment. The GIWAXS patterns were measured at an incident angle of $1^{\circ}$ at beamline 7.3.3 at the Advanced Light Source in Lawrence Berkeley National Laboratory with a $10 \mathrm{keV}$ $\mathrm{X}$-ray energy on a Pilatus $2 \mathrm{M}$ detector ${ }^{36}$. The patterns were corrected and produced using Igor Pro and a modified version of the NIKA package ${ }^{37}$. The color scale of the patterns is logarithmic. The molecular schematics in Supplementary Figure $3 \mathrm{~b}$ and Supplementary Figure $3 \mathrm{~d}$ were created using CrystalMaker ${ }^{\circledR}$ version 10.2.2. The layered perovskite $(\mathrm{BA})_{2}(\mathrm{MA})_{3} \mathrm{~Pb}_{4} \mathrm{I}_{13}$ thin films for $2 \mathrm{D}$ GIWAXS measurement were fabricated with the same method as those in the high PCE layered perovskite solar cells, except that silicon (100) substrates were used while with PTAA layer on it.

Transmission electron microscope characterization. The HRTEM samples were prepared by extracting the cross-section of the thin film fabricated by hot-casting. Transmission electron microscopy (TEM) lift-out samples were prepared via focused ion beam (FIB) polishing in a Helios NanoLab ${ }^{\text {tw }} 660$ SEM. The sample was extracted by FIB with accelerating voltage of $5 \mathrm{kV}$ and current of $1.2 \mathrm{nA}$. After the lamella was prepared, it was welded to a TEM copper grid by platinum. After that, it was gradually thinned to electron transparency with an ion beam of accelerating voltage of $5 \mathrm{kV}$ and gradually reducing current of $41 \mathrm{pA}, 15 \mathrm{pA}$ and $7 \mathrm{pA}$. The SEM accelerating voltage was kept at $5 \mathrm{kV}$ for the entire process. Once the sample preparation was complete, the lift-out sample was transferred to the TEM chamber immediately in less than 1 min of exposure to air. The TEM was carried out by FEI Tecnai OsirisTM with $200 \mathrm{kV}$ and $80 \mathrm{kV}$ beam voltage.

To investigate if the samples are free from FIB damages, we doctor bladed the $(\mathrm{BA})_{2} \mathrm{MA}_{3} \mathrm{~Pb}_{4} \mathrm{I}_{13}$ precursor solution directly on copper grid. In Supplementary Figure 9, it can be seen that without FIB process the same wide lattice structure also showed up. Thus, the FIB process-induced damage can be excluded. Besides, damages from the transmitted electron beam were also investigated (in Supplementary Figure 10). We found that the sample transits from crystalline to 
amorphous phase when it is exposed to electron beam longer than $5 \mathrm{~s}^{38}$. Thus, all TEM images were taken within $5 \mathrm{~s}$.

Transient absorption spectroscopy. Transient absorption experiments are conducted with a $45 \mathrm{fs}, 4 \mathrm{~mJ}$ Coherent Libra with a $1 \mathrm{kHz}$ repetition rate. The $570 \mathrm{~nm}$ pump beam is from a visible continuum generated by focusing $1.5 \mathrm{~mJ}$ of the 800 $\mathrm{nm}$ fundamental into a $4 \mathrm{~m}$ long tube. An all-reflective $4 \mathrm{~F}$ setup is utilized to filter the desired portion of the spectrum. The spectrally filtered pump pulses have $5 \mathrm{~nm}$ widths and $250 \mathrm{fs}$ durations. Continuum probe pulses are generated in a sapphire window and relayed to the sample with reflective optics. Signal detection is accomplished with a CMOS (complementary metal-oxide-semiconductor) array detector that is synchronized to the $1 \mathrm{kHz}$ repetition rate of the laser system.

TPC measurement. During this measurement, a nanosecond pulsed $\mathrm{N}_{2}$ laser was used to generate charge carriers close to the anode of the device that were driven toward the cathode by the built-in electric field, and the photocurrent was recorded by a $\mathrm{GHz}$ oscilloscope with an input resistor of $50 \Omega$. For the as-fabricated device with working area of about $8 \mathrm{~mm}^{2}$, the measured response time of the device was comparable to its RC time constant, i.e., $175 \mathrm{~ns}$ (Fig. 7c). This indicates that the intrinsic charge transit time across the device is smaller than the RC time constant, which is consistent with our previous reports. To eliminate the influence of RC time constant on the TPC signal, we gradually decreased the working area of the same device so that the device capacitance was reduced. It is found that the discrepancy between the device RC time constant and the TPC decay time is becoming larger when decreasing the device area, implying the charge transit time gradually dominates the TPC signal. Based on the response time of the smallest area device $(\tau)$, we can derive the carrier mobility in the vertical direction with the following expression:

$$
\mu=\frac{d^{2}}{V_{\mathrm{b}} \tau},
$$

where $d$ is the film thickness and $V_{\mathrm{b}}$ is the built-in potential.

\section{Data availability}

The data that support the plots within this paper are available from the corresponding author upon request.

Received: 13 August 2018 Accepted: 30 January 2019

Published online: 01 March 2019

\section{References}

1. Jeon, N. J. et al. Compositional engineering of perovskite materials for highperformance solar cells. Nature 517, 476-480 (2015).

2. Stranks, S. D. et al. Electron-hole diffusion lengths exceeding 1 micrometer in an organometal trihalide perovskite absorber. Science 342, 341-344 (2013).

3. Xing, G. et al. Long-range balanced electron- and hole-transport lengths in organic-inorganic $\mathrm{CH}_{3} \mathrm{NH}_{3} \mathrm{PbI}_{3}$. Science 342, 344-347 (2013).

4. NREL chart, https://www.nrel.gov/pv/cell-efficiency.html (National Renewable Energy Laboratory).

5. Li, B., Li, Y., Zheng, C., Gao, D. \& Huang, W. Advancements in the stability of perovskite solar cells: degradation mechanisms and improvement approaches. RSC Adv. 6, 38079-38091 (2016).

6. Quan, L. N. et al. Ligand-stabilized reduced-dimensionality perovskites. J. Am. Chem. Soc. 138, 2649-2655 (2016).

7. Smith, I. C., Hoke, E. T., Solis-Ibarra, D., McGehee, M. D. \& Karunadasa, H. I. A layered hybrid perovskite solar-cell absorber with enhanced moisture stability. Angew. Chem. Int. Ed. Engl. 53, 11232-11235 (2014).

8. Cao, D. H., Stoumpos, C. C., Farha, O. K., Hupp, J. T. \& Kanatzidis, M. G. 2D homologous perovskites as light-absorbing materials for solar cell applications. J. Am. Chem. Soc. 137, 7843-7850 (2015).

9. Tsai, H. et al. High-efficiency two-dimensional ruddlesden-popper perovskite solar cells. Nature 536, 312-316 (2016).

10. Zhang, X. et al. Stable high efficiency two-dimensional perovskite solar cells via cesium doping. Energy Environ. Sci. 10, 2095-2102 (2017).

11. Liu, J., Leng, J., Wu, K., Zhang, J. \& Jin, S. Observation of internal photoinduced electron and hole separation in hybrid two-dimentional perovskite films. J. Am. Chem. Soc. 139, 1432-1435 (2017).

12. Ishihara, T., Takahashi, J. \& Goto, T. Optical properties due to electronic transitions in two-dimensional semiconductors $\left(\mathrm{C}_{\mathrm{n}} \mathrm{H}_{2 \mathrm{n}+1} \mathrm{NH}_{3}\right)_{2} \mathrm{PbI}_{4}$. Phys. Rev. B 42, 11099-11107 (1990).

13. Pedesseau, L. et al. Advances and promises of layered halide hybrid perovskite semiconductors. ACS Nano 10, 9776-9786 (2016).
14. Muljarov, E. A., Tikhodeev, S. G., Gippius, N. A. \& Ishihara, T. Excitons in self-organized semiconductor/insulator superlattices: PbI-based perovskite compounds. Phys. Rev. B 51, 14370-14378 (1995).

15. Blancon, J.-C. et al. Extremely efficient internal exciton dissociation through edge states in layered 2D perovskites. Science 355, 1288-1292 (2017).

16. Qiu, J. et al. All-solid-state hybrid solar cells based on a new organometal halide perovskite sensitizer and one-dimensional $\mathrm{TiO}_{2}$ nanowire arrays. Nanoscale 5, 3245-3248 (2013).

17. Baikie, T. et al. Synthesis and crystal chemistry of the hybrid perovskite $\left(\mathrm{CH}_{3} \mathrm{NH}_{3}\right) \mathrm{PbI}_{3}$ for solid-state sensitised solar cell applications. J. Mater. Chem. A 1, 5628 (2013).

18. Stoumpos, C. C. et al. Ruddlesden-Popper hybrid lead iodide perovskite 2D homologous semiconductors. Chem. Mater. 28, 2852-2867 (2016).

19. Xiao, Z. et al. Efficient perovskite light-emitting diodes featuring nanometresized crystallites. Nat. Photonics 11, 108-115 (2017).

20. Lee, S. et al. Growth of nanosized single crystals for efficient perovskite lightemitting diodes. ACS Nano 12, 3417-3423 (2018).

21. Quintero-Bermudez, R. et al. Compositional and orientational control in metal halide perovskites of reduced dimensionality. Nat. Mater. 17, 900-907 (2018).

22. Chen, A. Z. et al. Origin of vertical orientation in two-dimensional metal halide perovskites and its effect on photovoltaic performance. Nat. Commun. 9, 1336 (2018).

23. Soe, C. M. M. et al. Understanding film formation morphology and orientation in high member 2D Ruddlesden-Popper perovskites for highefficiency solar cells. Adv. Energy Mater. 8, 1700979 (2018).

24. Williams, O. F. et al. Energy transfer mechanisms in layered 2D perovskites J. Chem. Phys. 148, 134706 (2018).

25. Yan, L. et al. General post-annealing method enables high-efficiency twodimensional perovskite solar cells. ACS Appl. Mater. Interfaces 10 33187-33197 (2018).

26. Coropceanu, V. et al. Charge transport in organic semiconductors. Chem. Rev 107, 926-952 (2007).

27. Mendaza, A. D. et al. High-entropy mixtures of pristine fullerenes for solutionprocessed transistors and solar cells. Adv. Mater. 27, 7325-7331 (2015).

28. Fishchuk, I. I. et al. Temperature dependence of the charge carrier mobility in disordered organic semiconductors at large carrier concentrations. Phys. Rev. B 81, 045202 (2010).

29. Milot, R. L., Eperon, G. E., Snaith, H. J., Johnston, M. B. \& Herz, L. M. Temperature-dependent charge-carrier dynamics in $\mathrm{CH}_{3} \mathrm{NH}_{3} \mathrm{PbI}_{3}$ perovskite thin films. Adv. Funct. Mater. 25, 6218-6227 (2015).

30. Shao, Y. et al. Grain boundary dominated ion migration in polycrystalline organic-inorganic halide perovskite films. Energy Environ. Sci. 9, 1752-1759 (2016).

31. Lin, Y. et al. Suppressed ion migration in low-dimensional perovskites. ACS Energy Lett. 2, 1571-1572 (2017).

32. Xiao, X. et al. Suppressed ion migration along the in-plane direction in layered perovskites. ACS Energy Lett. 3, 684-688 (2018).

33. Zhao, J. et al. Strained hybrid perovskite thin films and their impact on the intrinsic stability of perovskite solar cells. Sci. Adv. 3, eaao5616 (2017).

34. Depmeier, W. \& Chapuis, G. The crystal structure of Bis(n-butylammonium) tetraehloromanganate(II), $\left(\mathrm{n}-\mathrm{C}_{4} \mathrm{H}_{9} \mathrm{NH}_{3}\right)_{2} \mathrm{MnCl}_{4}$, at $294 \mathrm{~K}$. Acta Crystallogr. Sect. B 35, 1081-1084 (1979).

35. Wu, X., Trinh, M. T. \& Zhu, X. Y. Excitonic many-body interactions in twodimensional lead iodide perovskite quantum wells. J. Phys. Chem. C 119, 14714-14721 (2015)

36. Hexemer, A. et al. A SAXS/WAXS/GISAXS beamline with multilayer monochromator. J. Phys. Conf. Ser. 247, 012007 (2010).

37. Ilavsky, J. Nika: software for two-dimensional data reduction. J. Appl. Crystallogr. 45, 324-328 (2012)

38. Kim, T. W. et al. Self-organized superlattice and phase coexistence inside thin film organometal halide perovskite. Adv. Mater. 30, 1705230 (2018).

\section{Acknowledgements}

This work is supported by National Science Foundation (OIA-1538893 and DMR 1420645), Office of Naval Research under award N00014-17-1-2727 and University of North Carolina's Research Opportunities Initiative (UNC ROI) through Center of Hybrid Materials Enabled Electronic Technology. M.M.N. and H.A. are also supported by the same UNC ROI. The research was performed in part in the Nebraska Nanoscale Facility: National Nanotechnology Coordinated Infrastructure, the Nanoengineering Research Core Facility, and the Nebraska Center for Materials and Nanoscience, which are supported by the National Science Foundation under Award ECCS: 1542182 and the Nebraska Research Initiative. The GIWAXS measurement and analysis by S.J.S. are supported by the National Science Foundation (No. DGE-1633587). The GIWAXS data were acquired at beamline 7.3.3 at the Advanced Light Source (ALS) in Lawrence Berkeley National Laboratory, which is supported by the U.S. Department of Energy (No. DE-AC02-05CH11231). Experimental support was provided at the ALS by beamline scientists C. Zhu and E. Schaible. N.Z. and 
A.M.M. are supported by National Science Foundation grant CHE-1763207. Y.L. thanks the support from China Scholarship Council.

\section{Author contributions}

J.H. conceived the idea and supervised the project. Y.L. fabricated the photovoltaic devices, synthesized layered perovskite single crystals and conducted the related optical and electrical properties characterizations. Y.F. measured the carrier mobility of layered perovskite single crystals and thin films. J.Z. and J.E.S. conducted the TEM characterization. J.Z. conducted the strain analysis. S.J.S., M.M.N. and H.A. conducted the GIWAXS measurements and analyzed the data. Q.W. conducted absorption measurements. N.Z. and A.M.M. conducted the TA measurements and analyzed the data. Y.L., Y. F., J.Z., Y.S. and J.H. wrote the paper.

\section{Additional information}

Supplementary Information accompanies this paper at https://doi.org/10.1038/s41467019-08958-9.

Competing interests: The authors declare no competing interests.

Reprints and permission information is available online at http://npg.nature.com/ reprintsandpermissions/
Journal peer review information: Nature Communications thanks the anonymous reviewers for their contribution to the peer review of this work.

Publisher's note: Springer Nature remains neutral with regard to jurisdictional claims in published maps and institutional affiliations.

(c) (i) Open Access This article is licensed under a Creative Commons Attribution 4.0 International License, which permits use, sharing, adaptation, distribution and reproduction in any medium or format, as long as you give appropriate credit to the original author(s) and the source, provide a link to the Creative Commons license, and indicate if changes were made. The images or other third party material in this article are included in the article's Creative Commons license, unless indicated otherwise in a credit line to the material. If material is not included in the article's Creative Commons license and your intended use is not permitted by statutory regulation or exceeds the permitted use, you will need to obtain permission directly from the copyright holder. To view a copy of this license, visit http://creativecommons.org/ licenses/by/4.0/.

(C) The Author(s) 2019 